\title{
Evidence that particle acceleration in hotspots of FR II galaxies is not constrained by synchrotron cooling
}

\author{
Anabella T. Araudo ${ }^{\mathrm{a}, \mathrm{b}}$, Anthony R. Bell ${ }^{\mathrm{c}}$, Katherine M. Blundell ${ }^{\mathrm{b}}$ \\ ${ }^{a}$ Laboratoire Univers et Particules de Montpellier CNRS/Universit de Montpellier, Place E. Bataillon, 34095 Montpellier, France \\ ${ }^{b}$ University of Oxford, Astrophysics, Keble Road, Oxford OX1 3RH, UK \\ ${ }^{c}$ University of Oxford, Clarendon Laboratory, Parks Road, Oxford OX1 3PU, UK
}

\begin{abstract}
We study the hotspots of powerful radiogalaxies, where electrons accelerated at the jet termination shock emit synchrotron radiation. The turnover of the synchrotron spectrum is typically observed between infrared and optical frequencies, indicating that the maximum energy of non-thermal electrons accelerated at the shock is $\lesssim \mathrm{TeV}$ for a canonical magnetic field of $\sim 100 \mu \mathrm{G}$. We show that this maximum energy cannot be constrained by synchrotron losses as usually assumed, unless the jet density is unreasonably large and most of the jet upstream energy goes to non-thermal particles. We test this result by considering a sample of hotspots observed at radio, infrared and optical wavelengths.
\end{abstract}

Keywords: galaxies: active, galaxies: jets, acceleration of particles, radiation mechanisms: non-thermal, shock waves

\section{Introduction}

The jet termination region of Fanaroff-Riley (FR) radiogalaxies [1] is characterised by a double shock structure separated by a contact discontinuity, as sketched in Figure 11. Hotspots are the downstream region of the jet reverse shock, where electrons accelerated by the shock emit synchrotron radiation. The cut-off of the synchrotron spectrum at $v_{\mathrm{c}} \gtrsim 10^{14} \mathrm{~Hz}$ typically observed in hotspots [e.g. 3, 4, 5, 6, 7] indicates that the maximum energy of non-thermal electrons is

$$
\frac{E_{\mathrm{c}}}{\mathrm{TeV}} \sim 0.2\left(\frac{v_{\mathrm{c}}}{10^{14} \mathrm{~Hz}}\right)^{\frac{1}{2}}\left(\frac{B}{100 \mu \mathrm{G}}\right)^{-\frac{1}{2}},
$$

where $B$ is the magnetic field [8]. In some cases, $\mathrm{X}$ rays are also detected and modeled as synchrotron self Compton emission and Compton up-scattering of Cosmic Microwave Background photons [e.g. 9, 10].

\footnotetext{
${ }^{1}$ Note that the contact discontinuity is unstable due to the velocity shear and density contrast in both sides of the discontinuity [e.g. 2].
}

Ions can also be accelerated in the jet reverse shock. Given that hadronic losses are very slow in low density plasmas such as the termination region of AGN jets, protons might achieve energies as large as the limit imposed by the size of the source (i.e. the "Hillas" limit). In particular, mildly relativistic reverse shocks with velocity $v_{\mathrm{sh}} \sim c / 3$ [11, 12] might accelerate particles with Larmor radius $r_{\mathrm{g}} \sim R_{\mathrm{j}}$, where $R_{\mathrm{j}}$ is the jet width at the termination region. Particles with such a large $r_{\mathrm{g}}$ have energy

$$
\frac{E_{\mathrm{UHECR}}}{\mathrm{EeV}} \sim 100\left(\frac{v_{\mathrm{sh}}}{c / 3}\right)\left(\frac{B}{100 \mu \mathrm{G}}\right)\left(\frac{R_{\mathrm{j}}}{\mathrm{kpc}}\right)
$$

[13, 14], and therefore hotspots have been proposed as sources of Ultra High Energy Cosmic Rays (UHECRs) [e.g. 15, 16]. But, there are two assumptions in Eq. (2):

1. Particles diffuse in the Bohm regime, i.e. the meanfree path is $\lambda \sim r_{\mathrm{g}}$.

2. The magnetic field $B$ persists over distances $\sim R_{\mathrm{j}}$ downstream of the shock. 


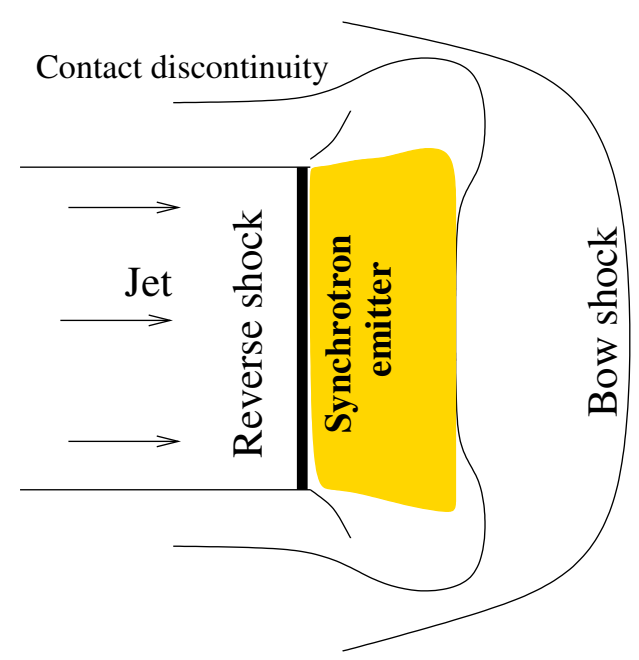

Figure 1: Sketch of the standard picture of the jet termination region. Particles are accelerated at the reverse shock, and radiate in the shock downstream region, here labelled "Synchrotron emitter".

Damping of the magnetic field in the southern hotspot of the quasar 4C74.26 was confirmed by modelling the compact synchrotron emission $(\sim 0.1 \mathrm{kpc})$ detected by the MERLIN interferometer at $1.66 \mathrm{GHz}$ [17]. The very thin synchrotron emitter would require a magnetic field $\sim 2.4 \mathrm{mG}$ to match the size of the emision region with the synchrotron cooling length at $1.66 \mathrm{GHz}$. This value of the magnetic field is about 10 times the upper limit imposed by the equipartition condition with nonthermal particles (see more details of the model in [18]). Therefore, the compact radio emission delineates the region within which the magnetic field is amplified up to $\sim 100 \mu \mathrm{G}$, and it is damped downstream of the shock. The cutoff of the synchrotron spectrum in the southern hotspot of 4C74.26 is between infrared (IR) and optical frequencies, and therefore the maximum energy of nonthermal electrons accelerated by the termination shock is $E_{\mathrm{c}} \sim 0.3 \mathrm{TeV}$. Given that the thickness of the MERLIN radio emitter is larger than the synchrotron cooling length of $0.3 \mathrm{TeV}$-electrons, we interpreted this behaviour of $E_{\mathrm{c}}$ being determined by synchrotron cooling, and then, at distances $\sim 0.1 \mathrm{kpc}$ downstream of the shock, the magnetic field is damped as a consequence of the small scale of Weibel turbulence. However, $0.1 \mathrm{kpc}$ is much larger than the turbulence decay length predicted by numerical simulations of Weibelmediated shocks in plasmas with densities $\sim 10^{-4} \mathrm{~cm}^{-3}$.

In a recent paper [19], we revisit the assumption of the cutoff of the hotspot synchrotron spectrum being determined by synchrotron losses. Given that the scalelength of magnetic fluctuations has to be larger than the plasma skin depth $c / \omega_{\mathrm{pi}}$, we show that $E_{\mathrm{c}}$ cannot be determined by synchrotron cooling, as usually assumed, unless very extreme conditions are assumed. We consider the sample of hotspots observed with high spatial resolution at radio, IR and optical frequencies in [20]. We also show that the Weibel instability is not the source of the amplified magnetic field throughout the whole hotspot emission region since not only does it damp too quickly, but also it generates turbulence on a very small scale, insufficient to accelerate particles up to $E_{\mathrm{c}} \sim \mathrm{TeV}$ for typical values of the magnetic field. We discuss the alternative possibility that the turbulence is generated by the Non Resonant Hybrid (NRH) instability [21, 22] which damps less quickly and grows on a larger scale. In the present contribution we highlight these results.

We remark that the results presented in [19] have important implications for Eq. (2) and the maximum energy that protons can achieve by being accelerated in the jet reverse shock. We conclude that hotspots of FR II radiogalaxies with IR/optical synchrotron cut-off are very poor accelerators of UHECRs.

\section{The reigning paradigm}

The traditional assumption is that $E_{\mathrm{c}}$ is determined by synchrotron cooling. By equating the synchrotron cooling timescale $t_{\text {synchr }} \sim 300 /\left(B^{2} E_{\mathrm{c}}\right)$ s with the acceleration time $t_{\mathrm{acc}} \sim 20 \mathcal{D} / v_{\mathrm{sh}}^{2}$, the diffusion coefficient of electrons with energy $E_{\mathrm{c}}$ (as in Eq. 1 is ${ }^{2}$

$$
\frac{\mathcal{D}_{\mathrm{c}, \mathrm{s}}}{\mathcal{D}_{\text {Bohm }}} \sim 10^{7}\left(\frac{v_{\mathrm{sh}}}{c / 3}\right)^{2}\left(\frac{v_{\mathrm{c}}}{10^{14} \mathrm{~Hz}}\right)^{-1},
$$

[e.g. 23, 24], where $\mathcal{D}_{\text {Bohm }} \sim r_{\mathrm{g}} c / 3$ is the Bohm diffusion coefficient. Note that protons with energy $\sim E_{\mathrm{c}}$ also diffuse with $\mathcal{D}_{\mathrm{c}, \mathrm{s}}$ and therefore the maximum energy that they can achieve is reduced to $10 \mathrm{TeV}$ instead of $100 \mathrm{EeV}$ as expected from the Hillas constraint in Eq. (2).

The mean-free path of particles diffusing in a medium with $\mathcal{D}_{\mathrm{c}, \mathrm{s}}$ is

$$
\lambda_{\mathrm{c}, \mathrm{s}} \sim \frac{\mathcal{D}_{\mathrm{c}, \mathrm{s}}}{c / 3} \sim 25\left(\frac{v_{\mathrm{sh}}}{c / 3}\right)^{2}\left(\frac{v_{\mathrm{c}}}{10^{14} \mathrm{~Hz}}\right)^{-\frac{1}{2}}\left(\frac{B}{100 \mu \mathrm{G}}\right)^{-\frac{3}{2}} \text { pc.(4) }
$$

We will show in the next section that $\lambda_{\mathrm{c}, \mathrm{s}}$ is larger than the maximum value of the mean-free path imposed by plasma physics.

\footnotetext{
${ }^{2}$ The subscript "c,s" in the diffusion coefficient $\mathcal{D}$ and in the meanfree path $\lambda$ means that the energy of particles is $E_{\mathrm{c}}$ and it is determined by synchrotron losses (i.e. $t_{\text {synchr }}\left(E_{\mathrm{c}}\right)=t_{\mathrm{acc}}\left(E_{\mathrm{c}}\right)$ ).
} 


\section{Revising the reigning paradigm}

The mean-free path of particles in a medium with (small) magnetic-turbulence scale-length $s$ is $\lambda \sim r_{\mathrm{g}}^{2} / s$ [e.g. 25, 24, 26, 27]. For a hydrogen plasma jet with proton thermal Lorentz factor $\sim 1$, density $n_{\mathrm{j}}$, and frequency $\omega_{\mathrm{pi}}$, the ion-skin depth downstream of the shock is

$$
\frac{c}{\omega_{\mathrm{pi}}} \sim 8.6 \times 10^{8}\left(\frac{n_{\mathrm{j}}}{10^{-4} \mathrm{~cm}^{-3}}\right)^{-\frac{1}{2}} \mathrm{~cm} .
$$

Considering that $s$ cannot be smaller than $c / \omega_{\mathrm{pi}}$, we find the upper-limit $\lambda_{\max }=r_{\mathrm{g}}^{2} /\left(c / \omega_{\mathrm{pi}}\right)$. In particular, $\lambda_{\max }$ of the most energetic electrons accelerated at the jet reverse shock is

$$
\begin{aligned}
\lambda_{\max } & =\frac{r_{\mathrm{g}, \mathrm{c}}^{2}}{c / \omega_{\mathrm{pi}}} \\
& \sim 0.02\left(\frac{v_{\mathrm{c}}}{10^{14} \mathrm{~Hz}}\right)\left(\frac{B}{100 \mu \mathrm{G}}\right)^{-3}\left(\frac{n_{\mathrm{j}}}{10^{-4} \mathrm{~cm}^{-3}}\right)^{\frac{1}{2}} \mathrm{pc},
\end{aligned}
$$

where

$$
\frac{r_{\mathrm{g}, \mathrm{c}}}{\mathrm{cm}} \sim 9 \times 10^{12}\left(\frac{v_{\mathrm{c}}}{10^{14} \mathrm{~Hz}}\right)^{0.5}\left(\frac{B}{100 \mu \mathrm{G}}\right)^{-1.5}
$$

is the Larmor radius of $E_{\mathrm{c}}$-electrons $\left(r_{\mathrm{g}, \mathrm{c}} \equiv r_{\mathrm{g}}\left(E_{\mathrm{c}}\right)\right)$. Therefore, the maximum diffusion coefficient is

$$
\begin{aligned}
\frac{\mathcal{D}_{\max }}{\mathcal{D}_{\text {Bohm }}}= & \frac{\lambda_{\max }}{r_{\mathrm{g}, \mathrm{c}}} \sim 3.2 \times 10^{4}\left(\frac{v_{\mathrm{c}}}{10^{14} \mathrm{~Hz}}\right)^{\frac{1}{2}} \\
& \left(\frac{B}{100 \mu \mathrm{G}}\right)^{-\frac{3}{2}}\left(\frac{n_{\mathrm{j}}}{10^{-4} \mathrm{~cm}^{-3}}\right)^{\frac{1}{2}} .
\end{aligned}
$$

If $E_{\mathrm{c}}$ were determined by a competition between shock acceleration and synchrotron cooling (the reigning paradigm), the mean-free path of $E_{\mathrm{c}}$-electrons would be given by Eq. (47) By comparing $\lambda_{\mathrm{c}, \mathrm{s}}$ with the upper-limit $\lambda_{\max }$, we find that

$$
\begin{gathered}
\frac{\lambda_{\mathrm{c}, \mathrm{s}}}{\lambda_{\max }} \sim 3 \times 10^{4}\left(\frac{v_{\mathrm{sh}}}{c / 3}\right)^{2}\left(\frac{v_{\mathrm{c}}}{10^{14} \mathrm{~Hz}}\right)^{-\frac{3}{2}}\left(\frac{B}{100 \mu \mathrm{G}}\right)^{\frac{3}{2}} \\
\left(\frac{n_{\mathrm{j}}}{10^{-4} \mathrm{~cm}^{-3}}\right)^{-\frac{1}{2}} .
\end{gathered}
$$

Setting $\lambda_{\mathrm{c}, \mathrm{s}} \leq \lambda_{\max }$ (or $\mathcal{D}_{\mathrm{c}, \mathrm{s}} \leq \mathcal{D}_{\max }$ ) implies a magnetic field $B \leq B_{\text {max }, \mathrm{s}}$, where

$$
\frac{B_{\mathrm{max}, \mathrm{s}}}{\mu \mathrm{G}} \sim 0.8\left(\frac{v_{\mathrm{c}}}{10^{14} \mathrm{~Hz}}\right)\left(\frac{v_{\mathrm{sh}}}{c / 3}\right)^{-\frac{4}{3}}\left(\frac{n_{\mathrm{j}}}{10^{-4} \mathrm{~cm}^{-3}}\right)^{\frac{1}{3}}
$$

In Fig. 2 we plot $B_{\max , \mathrm{s}}$ for the cases of $n_{\mathrm{j}}=10^{-4}$ (bluesolid line) and $10^{-6} \mathrm{~cm}^{-3}$ (blue-dashed line). The small

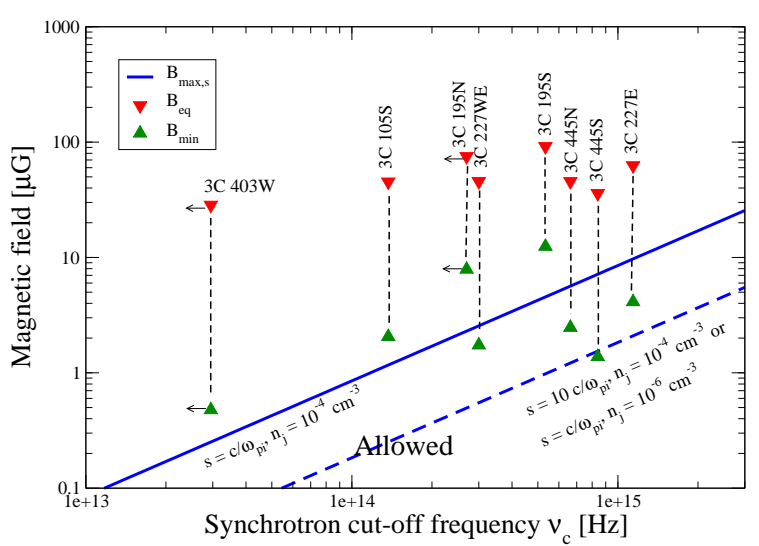

Figure 2: Upper limit $B_{\max , \mathrm{s}}$ for the magnetic field imposed by the condition $\lambda_{\mathrm{c}, \mathrm{s}} \geq \lambda_{\max }\left(n_{\mathrm{j}}=10^{-4} \mathrm{~cm}^{-3}\right.$ : blue-solid line; $n_{\mathrm{j}}=$ (6) $-6 \mathrm{~cm}^{-3}$ : blue-dashed line). Triangles indicate the maximum ( $B_{\mathrm{eq}}$, red triangles) and minimum ( $B_{\mathrm{min}}$, green triangles) field for the sources in Mack et al. (2009).

values of $B_{\max , \mathrm{s}}$ would require a very large energy density in non-thermal electrons $\left(U_{e}\right)$ in order to explain the measured radio synchrotron flux, as described in the next section. However, $U_{e}$ cannot be larger than the jet kinetic energy density $U_{\text {kin }}=m_{p} c^{3} n_{\mathrm{j}}\left(\Gamma_{\mathrm{j}}-1\right)$, where $\Gamma_{\mathrm{j}}=1.06$ is the bulk jet Lorentz factor if the jet velocity is $c / 3$ :

$$
\frac{U_{\text {kin }}}{\operatorname{erg~\mathrm {cm}^{-3}}} \sim 10^{-8}\left(\frac{\Gamma_{\text {jet }}-1}{0.06}\right)\left(\frac{n_{\mathrm{j}}}{10^{-4} \mathrm{~cm}^{-3}}\right) .
$$

\subsection{Synchrotron emission at $8.4 \mathrm{GHz}$}

We consider the sample of 8 hotspots observed at $8.4 \mathrm{GHz}$ by [20], and with radio-to-optical spectral in$\operatorname{dex} \alpha$ (see Table 11). Synchrotron emission is produced by non-thermal electrons following a power-law energy distribution $N_{e}=K_{e} E_{e}^{-p}$, where $E_{e} \geq m_{e} c^{2} \gamma_{\min }$ and $p=2 \alpha+1$. The normalization constant $K_{e}$ can be determined from the synchrotron luminosity at $8.4 \mathrm{GHz}$ $\left(L_{8.4}\right)$ emitted in a volume $V$ [see more details in 18], and $U_{e} \sim K_{e} \gamma_{\min }^{2-p} /(p-2)$ can be written as

$$
\begin{aligned}
\frac{U_{e}}{\operatorname{erg~cm} c^{-3}} & \sim 10^{-9}\left(\frac{p-2}{0.5}\right)^{-1}\left(\frac{\gamma_{\mathrm{min}}}{100}\right)^{2-p} \\
& \left(\frac{L_{8.4}}{10^{41} \mathrm{erg} \mathrm{s}^{-1}}\right)\left(\frac{V}{\mathrm{kpc}^{3}}\right)^{-1}\left(\frac{B}{100 \mu \mathrm{G}}\right)^{\frac{-p-1}{2}} .
\end{aligned}
$$

By setting the extreme condition $U_{e}=U_{\text {kin }}$ we find 
a lower limit in the magnetic field $\left(B_{\min }\right)$ needed to explain the synchrotron emission at $8.4 \mathrm{GHz}$

$$
\begin{aligned}
\frac{B_{\min }}{\mu \mathrm{G}} & \sim 27^{\frac{3.5}{p+1}}\left(\frac{\gamma_{\min }}{100}\right)^{\frac{4-2 p}{(p+1)}}\left(\frac{L_{8.4}}{10^{41} \mathrm{erg} \mathrm{s}^{-1}}\right)^{\frac{2}{p+1}} \\
& {\left[\left(\frac{\Gamma_{\mathrm{j}}-1}{0.06}\right)\left(\frac{p-2}{0.5}\right)\left(\frac{V}{\mathrm{kpc}^{3}}\right)\left(\frac{n_{\mathrm{j}}}{10^{-4} \mathrm{~cm}^{-3}}\right)\right]^{\frac{-2}{p+1}} . }
\end{aligned}
$$

We compute $B_{\min }$ for all the sources in [20]; see Table 1 ] and Fig. 2 (green-triangles up) where we also list and plot the value of the magnetic field $B_{\text {eq }}$ in equipartition with non-thermal electrons (i.e. $\left.B_{\mathrm{eq}}^{2} /(8 \pi)=U_{e}\right)$. We can see that $B_{\min }>B_{\max , \mathrm{s}}$ (blue-solid line) for sources $3 \mathrm{C} 105 \mathrm{~S}, 3 \mathrm{C} 195 \mathrm{~N}, 3 \mathrm{C} 195 \mathrm{~S}$ and $3 \mathrm{C} 403 \mathrm{~W}$ whereas $B_{\min }<B_{\text {max }, \mathrm{s}}$ for $3 \mathrm{C} 227 \mathrm{WE}, 3 \mathrm{C} 227 \mathrm{E}, 3 \mathrm{C} 445 \mathrm{~N}$ and $3 \mathrm{C} 445 \mathrm{~S}$. Note however that:

- The jet density $n_{\mathrm{j}} \sim 10^{-4} \mathrm{~cm}^{-3}$ is the upper limit found in Cygnus A and 3C475, and therefore we expect values of $B_{\min }$ greater than those plotted in Fig. 2 when the jet density is smaller than $10^{-4} \mathrm{~cm}^{-3}\left(B_{\min } \propto n_{\mathrm{j}}^{-(p+1) / 2}\right)$. On the other hand, $B_{\mathrm{max}, \mathrm{s}} \propto n_{\mathrm{j}}^{1 / 3}$ and therefore $B_{\mathrm{max}, \mathrm{s}}$ decreases when smaller values of $n_{\mathrm{j}}$ are considered and the ratio $B_{\min } / B_{\max , \mathrm{s}} \propto n_{\mathrm{j}}^{-(p+5 / 6)}$. In particular, the blue-dashed line in Fig. 2 corresponds to the case of $n_{\mathrm{j}}=10^{-6} \mathrm{~cm}^{-3}$ and $s=c / \omega_{\mathrm{pi}}$. In such a case, sources $3 \mathrm{C} 227 \mathrm{WE}, 3 \mathrm{C} 227 \mathrm{E}, 3 \mathrm{C} 445 \mathrm{~N}$ and $3 \mathrm{C} 445 \mathrm{~S}$ move to the regime where $B_{\min }>B_{\max , \mathrm{s}}$. The minimum value of the jet density required to match $B_{\min }=B_{\max , \mathrm{s}}$ is listed in Table 11 for all the sources considered in this paper. We can see for instance that the source 3C $195 \mathrm{~N}$ necessitates $n_{\mathrm{j}}>6.5 \times 10^{-4} \mathrm{~cm}^{-3}$ to satisfy the conditions $\lambda_{\mathrm{c}, \mathrm{s}}<\lambda_{\max }$ (i.e. $s>c / \omega_{\mathrm{pi}}$ ) and $U_{e}<U_{\text {kin }}$.

- Even when jets in FR galaxies are expected to be perpendicular to the line of sight, small departure from the plane of the sky (i.e. $\theta_{\mathrm{j}}<90^{\circ}$, where $\theta_{\mathrm{j}}$ is the angle between the jet and the line of sight) reduces the size of the shock downstream region. In such a case, $B_{\min } \propto V^{-2 /(p+1)}$ increases whereas $B_{\text {max,s }}$ remains constant. Therefore, the situation is even more strongly ruled out when $\theta_{\mathrm{j}}<90^{\circ}$.

Even in the case that the extreme conditions discussed before are assumed, the large value of the diffusion coefficient required to be $E_{\mathrm{c}}$ determined by synchrotron losses $\left(\mathcal{D}_{\mathrm{c}, \mathrm{s}} / \mathcal{D}_{\text {Bohm }} \sim 10^{6}-10^{7}\right.$, see Eq. (3) cannot be explained in any well-established theoretical framework [see Section 4 in 19]. We therefore suggest that the IR/optical cutoff in the synchrotron spectrum has a different cause.

\section{Particle acceleration and magnetic field amplifi- cation in quasi-perpendicular shocks}

If synchrotron losses are not relevant, the maximum energy is ultimately determined by the ability to scatter particles downstream of the shock. We consider that the amplified hotspot magnetic field $B$ is turbulent, and that the large scale background field downstream of the reverse shock $\left(B_{\mathrm{jd}}\right)$ is nearly perpendicular to the shock normal.

\subsection{Diffusive shock acceleration in quasi- perpendicular shocks}

To accelerate particles up to an energy $E_{\mathrm{c}}$ via a diffusive mechanism in a perpendicular shock, the mean-free path $\lambda_{\mathrm{c}} \sim r_{\mathrm{g}, \mathrm{c}}(B)^{2} / s$ in the shock downstream region has to be smaller than Larmor radius in $B_{\text {jd }}$ [e.g. 24, 26, 28]. Otherwise, the particles are carried away downstream by the magnetic field and acceleration is halted. The condition $\lambda_{\mathrm{c}} \leq r_{\mathrm{g}, \mathrm{c}}\left(B_{\mathrm{jd}}\right)$ is satisfied when the magneticturbulence scale-length is $s \geq s_{\perp}$, where

$$
\begin{aligned}
s_{\perp} & \equiv \frac{E_{\mathrm{c}}}{e B}\left(\frac{B_{\mathrm{jd}}}{B}\right) \\
& =5 \times 10^{11}\left(\frac{v_{\mathrm{c}}}{10^{14} \mathrm{~Hz}}\right)^{\frac{1}{2}}\left(\frac{B_{\mathrm{jd}}}{\mu \mathrm{G}}\right)\left(\frac{B}{100 \mu \mathrm{G}}\right)^{-\frac{5}{2}} \mathrm{~cm},
\end{aligned}
$$

and $s_{\perp}$ is the Larmor radius of protons (and electrons) with energy

$$
\begin{aligned}
E_{\mathrm{S}_{\perp}} & =E_{\mathrm{c}}\left(\frac{B_{\mathrm{jd}}}{B}\right)=0.07 E_{\mathrm{c}}\left(\frac{B_{\mathrm{j}}}{\mu \mathrm{G}}\right)\left(\frac{B}{100 \mu \mathrm{G}}\right)^{-1} \\
& \sim 10\left(\frac{v_{\mathrm{c}}}{10^{14} \mathrm{~Hz}}\right)^{\frac{1}{2}}\left(\frac{B_{\mathrm{jd}}}{\mu \mathrm{G}}\right)\left(\frac{B}{100 \mu \mathrm{G}}\right)^{-\frac{5}{2}} \mathrm{GeV}
\end{aligned}
$$

in a magnetic field $B$ (i.e. $s_{\perp}=E_{\mathrm{S}_{\perp}} / q B$ ). Note that $s_{\perp}$ in Eq. (14) is greater than $c / \omega_{\text {pi }}$ in Eq. (5), as required. However, $s>s_{\perp} \sim 500 c / \omega_{\text {pi }}$ (for typical values considered in this paper) cannot be fulfilled by Weibelgenerated turbulence with scale $\sim c / \omega_{\text {pi }}$.

\subsection{Non-resonant hybrid instabilities in quasi- perpendicular shocks}

Turbulence on a scale greater than $c / \omega_{\text {pi }}$ may be excited through the NRH instability [21, 22]. In the simplest form of it, the cosmic ray (CR) Larmor radius in the unperturbed background field is much greater than the wavelength of field perturbations and therefore the streaming of CRs carrying the electric current $j_{\mathrm{cr}}$ is undeflected. The force $-\vec{j}_{\text {cr }} \times \vec{B}$ acts to expand loops in the magnetic field, and therefore $B$ increases. This produces an increment in $-\vec{j}_{\text {cr }} \times \vec{B}$ and generates a positive 
Table 1: Physical parameters of the sources considered in this paper. The synchrotron cut-off frequency $\left(v_{\mathrm{c}}\right)$ and spectral index $(\alpha)$ are taken from Mack et al. (2009); and $p=2 \alpha+1$. The synchrotron luminosity at $8.4 \mathrm{GHz}$ is calculated as $L_{8.4}=S_{8.4} 8.4 \times 10^{9}$, where $S_{8.4}$ is the measured flux. The hotspot volume $V$ is calculated from the angular sizes tabulated in Table 5 in Mack et al. (2009) together with $S_{8.4}$. The magnetic field in equipartition with non thermal electrons $\left(B_{\mathrm{eq}}\right)$ is calculated in Araudo et al. (2016) and it is similar to the values given in Mack et al. (2009). $B_{\text {min }}$ is the magnetic field required to emit $L_{8.4}$ when $U_{e}=U_{\mathrm{kin}}$, and $B_{\max , \mathrm{s}}$ is the magnetic field that satisfies the condition $\lambda_{\mathrm{c}, \mathrm{s}}=\lambda_{\mathrm{max}}$.

\begin{tabular}{lcc|ccccccc}
\hline Source & $\begin{array}{c}v_{\mathrm{c}} \\
{\left[10^{14} \mathrm{~Hz}\right]}\end{array}$ & $\alpha$ & $p$ & $\begin{array}{c}L_{8.4} \\
{[\mathrm{erg} / \mathrm{s}]}\end{array}$ & $\begin{array}{c}V \\
{\left[\mathrm{kpc}^{3}\right]}\end{array}$ & $\begin{array}{c}B_{\mathrm{eq}} \\
{[\mu \mathrm{G}]}\end{array}$ & $\begin{array}{c}B_{\min } \\
{[\mu \mathrm{G}]}\end{array}$ & $\begin{array}{c}B_{\mathrm{max}, \mathrm{s}} \\
{[\mu \mathrm{G}]}\end{array}$ & $\begin{array}{c}n_{\mathrm{j}, \min } \\
{\left[\mathrm{cm}^{-3}\right]}\end{array}$ \\
\hline 3C 105S & 1.37 & 0.75 & 2.5 & $1.42 \times 10^{42}$ & 1205.63 & 45.27 & 2.06 & 1.16 & $1.92 \times 10^{-4}$ \\
3C 195N & $<2.70$ & 0.95 & 2.9 & $1.15 \times 10^{41}$ & 38.12 & 75.11 & 7.89 & 2.30 & $6.51 \times 10^{-4}$ \\
3C 195S & 5.34 & 1.00 & 3.0 & $1.71 \times 10^{41}$ & 33.58 & 91.76 & 12.45 & 4.55 & $3.42 \times 10^{-4}$ \\
3C 227WE & 3.00 & 0.65 & 2.3 & $3.19 \times 10^{40}$ & 19.26 & 45.63 & 1.74 & 2.55 & $6.78 \times 10^{-5}$ \\
3C 227E & 11.4 & 0.75 & 2.5 & $7.14 \times 10^{40}$ & 17.99 & 62.60 & 4.12 & 9.71 & $3.96 \times 10^{-5}$ \\
3C 403W & $<0.29$ & 0.55 & 2.1 & $3.95 \times 10^{40}$ & 167.9 & 28.46 & 0.48 & 0.25 & $1.96 \times 10^{-4}$ \\
3C 445N & 6.63 & 0.85 & 2.7 & $2.18 \times 10^{40}$ & 29.36 & 45.60 & 2.47 & 5.65 & $3.97 \times 10^{-5}$ \\
3C 445S & 8.40 & 0.80 & 2.6 & $5.04 \times 10^{40}$ & 139.42 & 35.94 & 1.38 & 7.15 & $1.60 \times 10^{-5}$ \\
\hline \hline
\end{tabular}

feedback loop that drives the NRH instability and amplifies the magnetic field. For the diamagnetic drift in the plane of the shock to amplify the magnetic field, the $\mathrm{NRH}$ growth rate has to be sufficient for the instability to grow through $\sim 10$ e-foldings at the maximum growth rate $\Gamma_{\max }$ [21, 22, 29] in the time $t_{\perp} \sim r_{\mathrm{g}}\left(B_{\mathrm{js}}\right) / v_{\mathrm{d}}$ the plasma flows through a distance $r_{\mathrm{g}}\left(B_{\mathrm{js}}\right)$ in the downstream region at velocity $v_{\mathrm{d}} \sim v_{\mathrm{sh}} / 4$, where $r_{\mathrm{g}}\left(B_{\mathrm{js}}\right)$ is the Larmor radius in the ordered field $B_{\mathrm{js}}$. That is, the condition $\Gamma_{\max } t_{\perp}>10$ must be satisfied. If the field is strongly amplified, the instability can be expected to saturate when its characteristic scale grows to the Larmor radius of the CR driving the instability. Thus, $s_{\perp}$ in Eq. (14) can be expected to match the Larmor radius of the highest energy CR driving the instability. If these CR have an energy $E_{\text {nrh }}$, then $E_{\mathrm{nrh}} \sim E_{\mathrm{s}_{\perp}}$.

In order to check that there is enough energy in $E_{\text {nrh- }}$ protons to excite the non-resonant turbulence ${ }^{3}$. we consider whether the number of e-foldings required to amplify the magnetic field up to the saturation value is of the order of 10 [21, 22]. The condition $\Gamma_{\max } t_{\perp}>10$ leads to

$$
\eta \gtrsim 0.04\left(\frac{B_{\mathrm{j}}}{\mu \mathrm{G}}\right)\left(\frac{\Gamma_{\mathrm{j}}-1}{0.06}\right)^{-\frac{1}{2}}\left(\frac{n_{\mathrm{j}}}{10^{-4} \mathrm{~cm}^{-3}}\right)^{-\frac{1}{2}},
$$

where $\eta$ is the acceleration efficiency. Given that particles accelerated in relativistic shocks follow a powerlaw energy distribution steeper than the canonical distribution (i.e. $p>2$ as is clear in Table 1), the CR pressure is dominated by low energy particles. Therefore, the condition for NRH instability growth is that the acceleration efficiency of low energy CR has to be $\eta \sim 0.04$

\footnotetext{
${ }^{3}$ Note that $E_{\mathrm{S}_{\perp}} \sim 100 m_{p} v_{\mathrm{sh}}^{2}$ if $v_{\mathrm{sh}} \sim c / 3$ and therefore $E_{\mathrm{nrh}}-\mathrm{CRs}$ are mildly supra-thermal protons.
}

for characteristic values considered in this paper. Such a value of $\eta$ is very reasonable.

From these estimations we can conclude that NRH instabilities generated by CRs with energies $\sim E_{\text {nrh }}$ can grow fast enough to amplify the jet magnetic field from $\sim 1$ to $100 \mu \mathrm{G}$ and accelerate particles up to energies $E_{\mathrm{c}} \sim 0.2 \mathrm{TeV}$ observed in the hotspots of FR II radiogalaxies. The advantage of magnetic turbulence being generated by CRs current is that the amplified magnetic field persists over long distances downstream of the shock, and therefore particles accelerated very near the shock can emit synchrotron radiation far downstream.

\section{Summary and conclusions}

We have investigated the physical mechanism that constraints the maximum energy of particles accelerated at the jet reverse shock in FR II radiogalaxies.

By equating the acceleration and synchrotron cooling timescales, the mean free path $\lambda_{\mathrm{c}, \mathrm{s}}$ of $E_{\mathrm{c}}$-electrons is greater than the maximum value $\lambda_{\max }=r_{\mathrm{g}, \mathrm{c}}^{2} /\left(c / \omega_{\mathrm{pi}}\right)$ for reasonable values of the magnetic field and jet density (see Eq. (9)). By considering a sample of 8 hotspots observed at optical, IR and radio wavelengths [20], we show that unreasonable large values of the jet density would be required (see $n_{\mathrm{j}, \mathrm{min}}$ in Table 1 ) to explain the synchrotron flux at $8.4 \mathrm{GHz}$ if $E_{\mathrm{c}}$ were determined by synchrotron cooling (see Fig. 2).

If synchrotron losses are not relevant, the maximum energy is ultimately determined by the ability to scatter particles downstream of the shock. Weibel-mediated shocks generate the magnetic field and accelerate particles [e.g. 30, 31]. However, the characteristic scale of Weibel turbulence cannot account for the cut-off of the synchrotron spectrum observed in hotspots, nor the 
large extension of the hotspot synchrotron emission, much larger than the magnetic decay of $\sim 100 c / \omega_{\text {pi }}$ predicted by numerical simulations. A viable alternative is that turbulence is generated by the streaming of CRs with energy $E_{\mathrm{nrh}} \sim E_{\mathrm{c}} B_{\mathrm{jd}} / B \sim 0.01 E_{\mathrm{c}}$ (see Sect. 4). The amplified magnetic field has a scale-length of the order of the Larmor radius of $E_{\mathrm{nrh}}$-protons and persists over long distances downstream of the shock, accounting for the extension of the synchrotron emitter.

In a work in progress, we apply our arguments to the very well known source Cygnus A for which well resolved and multi-wavelength data are available [32]. We will show that the primary hotspot in the western jet of Cygnus A is a clear case where the maximum energy of electrons accelerated in the jet reverse shock is not constrained by synchrotron cooling.

\section{Acknowledgements}

A.T.A. thanks the organisers of the conference for their kind hospitality. The research leading to this article has received funding from the European Research Council under the European Community's Seventh Framework Programme (FP7/2007-2013)/ERC grant agreement no. 247039. We acknowledge support from the UK Science and Technology Facilities Council under grant No. ST/K00106X/1.

\section{References}

[1] B. L. Fanaroff, J. M. Riley, The morphology of extragalactic radio sources of high and low luminosity, MNRAS167 (1974) 31P-36P.

[2] A. Mizuta, S. Yamada, H. Takabe, Propagation and Dynamics of Relativistic Jets, ApJ606 (2004) 804-818. arXiv:astro-ph/ 0402355 doi: $10.1086 / 382779$

[3] K. Meisenheimer, A. F. Heavens, Particle acceleration in the hotspot of the jet of quasar 3C273, Nature323 (1986) 419-422. doi:10.1038/323419a0

[4] K. Meisenheimer, M. G. Yates, H.-J. Roeser, The synchrotron spectra of radio hot spots. II. Infrared imaging., A\&A325 (1997) 57-73.

[5] F. Tavecchio, R. Cerutti, L. Maraschi, R. M. Sambruna, J. K. Gambill, C. C. Cheung, C. M. Urry, X-Ray and Optical Emission from Radio Hot Spots of Powerful Quasars, ApJ630 (2005) 721-728. doi:10.1086/432371

[6] Ł. Stawarz, C. C. Cheung, D. E. Harris, M. Ostrowski, The Electron Energy Distribution in the Hotspots of Cygnus A: Filling the Gap with the Spitzer Space Telescope, ApJ662 (2007) 213223. arXiv: astro-ph/0701568 doi:10.1086/517966

[7] M. W. Werner, D. W. Murphy, J. H. Livingston, V. Gorjian, D. L. Jones, D. L. Meier, C. R. Lawrence, Spitzer Observations of Hotspots in Radio Lobes, ApJ759 (2012) 86. arXiv:1209. 0810 doi: $10.1088 / 0004-637 X / 759 / 2 / 86$

[8] V. L. Ginzburg, S. I. Syrovatskii, The Origin of Cosmic Rays, 1964.
[9] E. S. Perlman, M. Georganopoulos, E. M. May, D. Kazanas, Chandra Observations of the Radio Galaxy 3C 445 and the Hot Spot X-Ray Emission Mechanism, ApJ708 (2010) 1-8. arXiv: 0910.3021 doi:10.1088/0004-637X/708/1/1

[10] A. S. Wilson, A. J. Young, P. L. Shopbell, Chandra Observations of Cygnus A: Magnetic Field Strengths in the Hot Spots of a Radio Galaxy, ApJ544 (2000) L27-L30. arXiv:astro-ph/ 0009308 doi:10.1086/317293

[11] F. Casse, A. Marcowith, Astroparticle yield and transport from extragalactic jet terminal shocks, Astroparticle Physics 23 (2005) 31-56. arXiv:astro-ph/0411395 doi:10.1016/ j.astropartphys.2004.11.003

[12] K. C. Steenbrugge, K. M. Blundell, Multiwavelength study of CygnusA - I. Precession and slow jet speeds from radio observations, MNRAS388 (2008) 1457-1464. arXiv:0805.2169. doi:10.1111/j.1365-2966.2007.12665.x

[13] P. O. Lagage, C. J. Cesarsky, The maximum energy of cosmic rays accelerated by supernova shocks, A\&A125 (1983) 249257.

[14] A. M. Hillas, The Origin of Ultra-High-Energy Cosmic Rays, ARA\&A22 (1984) 425-444. doi : 10.1146/annurev .aa. 22. 090184.002233

[15] J. P. Rachen, P. L. Biermann, Extragalactic Ultra-High Energy Cosmic-Rays - Part One - Contribution from Hot Spots in FrII Radio Galaxies, A\&A272 (1993) 161. arXiv:astro-ph/ 9301010

[16] C. A. Norman, D. B. Melrose, A. Achterberg, The Origin of Cosmic Rays above 1018.5 eV, ApJ454 (1995) 60. doi:10. $1086 / 176465$

[17] A. T. Araudo, A. R. Bell, K. M. Blundell, Particle Acceleration and Magnetic Field Amplification in the Jets of 4C74.26, ApJ806 (2015) 243. arXiv:1505.02210 doi:10.1088/ 0004-637X/806/2/243

[18] A. T. Araudo, A. R. Bell, K. M. Blundell, Particle acceleration and magnetic field amplification in hotspots of FR II galaxies: The case study 4C74.26, ArXiv e-prints arXiv: 1602.07896

[19] A. T. Araudo, A. R. Bell, A. Crilly, K. M. Blundell, Evidence that the maximum electron energy in hotspots of FR II galaxies is not determined by synchrotron cooling, MNRAS460 (2016) 3554-3562. arXiv:1605.05125 doi:10. 1093/mnras/stw1204

[20] K.-H. Mack, M. A. Prieto, G. Brunetti, M. Orienti, Nearinfrared/optical counterparts of hotspots in radio galaxies, MNRAS392 (2009) 705-717. arXiv:0810.3764 doi:10.1111/ j.1365-2966.2008.14081.x

[21] A. R. Bell, Turbulent amplification of magnetic field and diffusive shock acceleration of cosmic rays, MNRAS353 (2004) 550-558. doi:10.1111/j.1365-2966.2004.08097.x

[22] A. R. Bell, The interaction of cosmic rays and magnetized plasma, MNRAS358 (2005) 181-187. doi:10.1111/j. 1365-2966.2005.08774.x

[23] M. D. Stage, G. E. Allen, J. C. Houck, J. E. Davis, Cosmicray diffusion near the Bohm limit in the Cassiopeia A supernova remnant, Nature Physics 2 (2006) 614-619. arXiv : astro-ph/ 0608401 doi:10.1038/nphys391

[24] J. G. Kirk, B. Reville, Radiative Signatures of Relativistic Shocks, ApJ710 (2010) L16-L20. arXiv:1001.0687 doi: 10.1088/2041-8205/710/1/L16

[25] M. Ostrowski, J. Bednarz, Comment on the first-order Fermi acceleration at ultra-relativistic shocks, A\&A394 (2002) 1141-1144. arXiv:astro-ph/0101069 doi:10.1051/ 0004-6361:20021173

[26] M. Lemoine, G. Pelletier, On electromagnetic instabilities at ultra-relativistic shock waves, MNRAS402 (2010) 321334. arXiv:0904.2657 doi:10.1111/j.1365-2966. 
2009.15869.x

[27] L. Sironi, A. Spitkovsky, J. Arons, The Maximum Energy of Accelerated Particles in Relativistic Collisionless Shocks, ApJ771 (2013) 54. arXiv:1301.5333 doi:10.1088/0004-637X/ $771 / 1 / 54$

[28] B. Reville, A. R. Bell, On the maximum energy of shockaccelerated cosmic rays at ultra-relativistic shocks, MNRAS439 (2014) 2050-2059. arXiv:1401.2803 doi:10.1093/ mnras/stu088

[29] A. R. Bell, Particle Acceleration by Shocks in Supernova Remnants, Brazilian Journal of Physics 44 (2014) 415-425. arXiv : 1311.5779 doi:10.1007/s13538-014-0219-5

[30] A. Spitkovsky, Particle Acceleration in Relativistic Collisionless Shocks: Fermi Process at Last?, ApJ682 (2008) L5-L8. arXiv:0802.3216 doi:10.1086/590248

[31] S. F. Martins, R. A. Fonseca, L. O. Silva, W. B. Mori, Ion Dynamics and Acceleration in Relativistic Shocks, ApJ695 (2009) L189-L193. arXiv:0903.3573 doi:10.1088/0004-637X/ 695/2/L189

[32] S. Pyrzas, K. C. Steenbrugge, K. M. Blundell, Multiwavelength study of Cygnus A. V. The hotspots in the lobe, A\&A574 (2015) A30. doi:10.1051/0004-6361/201425061 